\title{
Positional cloning and characterisation of the human DLGAP2 gene and its exclusion in progressive epilepsy with mental retardation
}

\author{
Susanna Ranta ${ }^{1,2}$, Yonghui Zhang ${ }^{3}$, Barbara Ross ${ }^{3}$, Elina Takkunen ${ }^{1,2}$, Aune Hirvasniemi ${ }^{4}$, \\ Albert de la Chapelle ${ }^{1,5}$, T Conrad Gilliam ${ }^{3}$ and Anna-Elina Lehesjoki ${ }^{1,2}$ \\ ${ }^{1}$ Folkhälsan Institute of Genetics, Helsinki; ${ }^{2}$ D epartment of Medical Genetics, University of Helsinki, Finland; \\ ${ }^{3}$ Columbia Genome Center, Columbia University, New York, USA; ${ }^{4}$ Department of Pediatrics, Kainuu Central \\ Hospital, Kajaani, Finland; ${ }^{5}$ Comprehensive Cancer Center, Ohio State University, Columbus, OH, USA
}

In search of the gene for progressive epilepsy with mental retardation (EPMR) we identified DLGAP2, the human homolog of the gene encoding the rat PSD-95/SAP90-associated protein-2 (Dlgap2). We extended the transcript in both the $5^{\prime}$ and $3^{\prime}$ directions and characterised the genomic structure of the approximately $10 \mathrm{~kb}$ gene. Sequence comparisons of human DLGAP2 cDNA sequences obtained from human testis and brain CDNA libraries with homologous rat genes suggest alternative splicing in the $5^{\prime}$ end of the gene. The $5^{\prime}$ coding sequence of the testis CDNA is complete, whereas based on homology with the rat gene 103 bp of coding sequence may still be missing in the $5^{\prime}$ end of the DLGAP2 brain transcript. DLGAP2 was excluded as the gene responsible for EPMR. European Journal of Human Genetics (2000) 8, 381-384.

Keywords: human DLGAP2 gene; exon-intron structure; progressive epilepsy with mental retardation; neuronal ceroid lipofuscinosis

Progressive epilepsy with mental retardation (EPMR) is an autosomal recessive disorder characterised by normal early development and childhood onset epilepsy followed by mental retardation. ${ }^{1}$ Recently, EPMR was recognised as a new subtype of neuronal ceroid lipofuscinoses (NCLs). ${ }^{2}$

We previously assigned the EPMR locus to an approximately $700 \mathrm{~kb}$ interval on chromosome $8 \mathrm{p}$ (Figure 1$){ }^{3-5}$ and constructed a $3412 \mathrm{bp}$ partial cDNA sequence (Genbank AF009204) mapping to this region. ${ }^{5}$ Between nucleotides 1259 and 2956 our cDNA sequence was identical to a partial cDNA sequence encoding a human discs large ( $\mathrm{hDLG}$ ) and a postsynaptic density protein $95 \mathrm{kDa}$ (PSD-95)-associated protein-2, DAP2 ${ }^{6}$ (GenBank AB000275), except for a $G$ to $A$ difference at position 1741 and 42 nucleotides downstream of position 1867 which was present in DAP2 but absent in our sequence. Our cDNA also showed homology to a rat gene

Correspondence: Susanna Ranta, Folkhälsan Institute of Genetics, Mannerheimintie 97, 00280 Helsinki, Finland. Tel: + 358961585558 ; Fax: +358961585632; E-mail: susanna.ranta@helsinki.fi Received 4 June 1999; revised 12 October 1999; accepted 19 October 1999
(GenBank U67138) encoding the PSD-95/synapse associated protein 90 (SAP90)-associated mRNA-2, Sapap2. ${ }^{7}$ According to the HUGO Nomenclature Committee, the DAP/SAPAP/ GKAP family of discs large associated proteins is denoted as DLGAP. In this paper we refer to the human homolog of the rat Sapap2 (DIgap2) gene as DLGAP2. The DLGAP proteins interact with the PSD-95/SAP90 protein, which in turn interacts with shaker type potassium channels and other molecules and clusters with these at synaptic junctions. ${ }^{6-8}$ PSD-95/SAP90 may play an important role in the organisation of ion channels and signalling molecules in the synaptic junction. Furthermore, as mutations in two potassium channel genes were recently identified underlying human epilepsy, ${ }^{9-11}$ we considered DLGAP2 a good positional candidate for EPMR.

The 3412 bp DLGAP2 sequence was first extended at the $3^{\prime}$ end by $1.4 \mathrm{~kb}$ using rapid extension of CDNA ends (RACE) (primer 5'-GTG GCCTGG CTCACACTT GGC TCT-3'; Clontech Marathon Ready Human Fetal Brain CDNA Library). Secondly, a brain specific EST ${ }^{12}$ (A004F35) was found to be part of the 3' untranslated region of DLGAP2. This EST was 


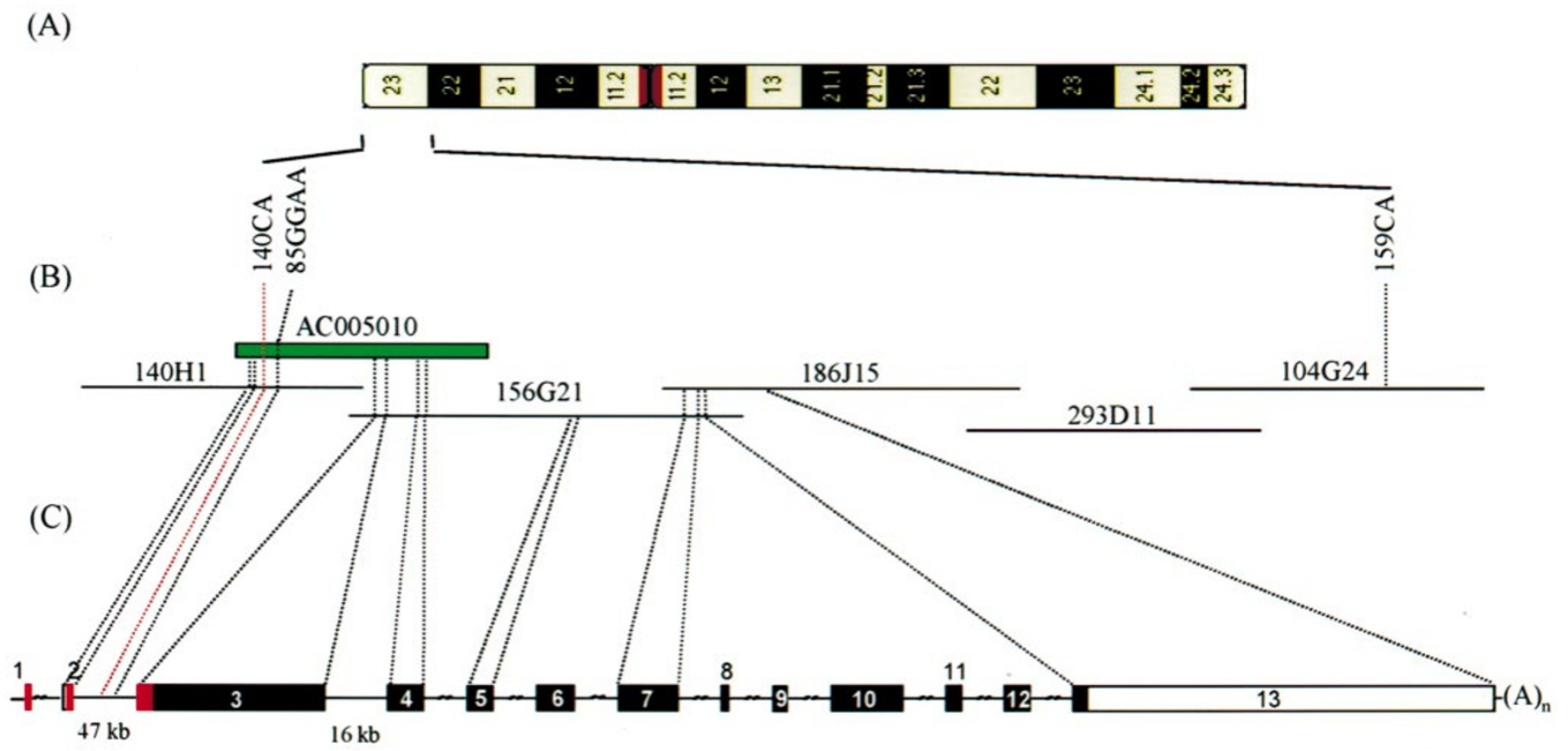

Figure 1 Positional cloning of DLGAP2 (A) The EPM R locus is located on chromosome 8p23 close to the telomere. (B) The EPM R critical region flanked by markers 140CA and 159CA is covered by five overlapping BAC clones. The green rectangle represents the sequence derived from GenBank (AC005010). (C) Genomic structure of DLGAP2. In brain, exons2-12 as well as the region of exon 13 marked with black is contained in the open reading frame. The CDNA sequence 5' to exon 2 is not known in brain, but resides outside the critical region. The coding region of the testis CDNA is marked in black. The 5 ' untranslated region of the testic cDNA is marked in red. Dotted lines indicate the location of the exons in BACs. Broken intronic lines indicate unknown intron sizes. The exon sizes are drawn approximately to scale except for the first and last exons. Exon 8 is not present in the cDNA clones identified by us.

extended by screening a brain cDNA library (Clontech cat. no. H13002a), by 5' RACE (primer 5'-TGG AAG GTC GGG GGATGAGTTG-3'; Clontech Marathon Ready Human Brain CDNA Library), and by incorporating sequences derived from three genomic clones from the EPM R region yielding a $3.2 \mathrm{~kb}$ sequence around this EST.

Telomeric to the 3412 bp sequence one exon obtained by exon amplification from BAC $140 \mathrm{H} 1$ (Figure 1$)^{5}$ was found homologous to the $5^{\prime}$ coding region of the rat DIgap2 (GenBank U67138). To confirm that this exon belonged to DLGAP2 we screened the Marathon Ready Brain CDNA Library with primers designed from the exon and DLGAP2 (5'-AGA AGC TGG AGACTTGGTCCAG-3' and 5'CTCAGC AGG TAG GGC GGC CG-3', nested primers 5'-AGC CGG GCATCA GCTTTC-3' and 5'-TCG GGC CCG TGG TGC AGGT-3'). A $214 \mathrm{bp}$ fragment bridging the exon to the known cDNA sequence was amplified. Further sequence comparison with the rat DIgap2 sequence suggested that $103 \mathrm{bp}$ of the $5^{\prime}$ coding sequence was still missing from DLGAP2. Of note, this putative missing region was found to reside outside the critical EPMR region (see below and Figure1).

We attempted to isolate the remaining 5 ' end by screening various RACE and CDNA libraries. Amplification of the
Marathon Ready Human Testis cDNA library (Clontech; primer 5'AGTAGC GCT CCT CATTGAAGT GCT GC-3'; nested primer 5'-TAC TGC GGG TCTAGA TCC TCC TCT G-3') resulted in a CDNA fragment containing a putative translation initiator ATG codon and an in-frame stop codon 5 ' to it. This ATG observed in the testis cDNA corresponds to the suggested start codon in the four known rat DIgap genes. However, in the rat DIgap2 this codon is the second ATG in the open reading frame. ${ }^{7}$ As in the human DLGAP2 the sequence derived from a brain library the sequence also remains open $5^{\prime}$ to the suggested initiator codon, and is homologous to rat DIgap2, it remains possible that in brain the translation of DLGAP2 is initiated at the earlier ATG than in other tissues. However, despite several attempts we were not able to isolate this longer brain DLGAP2 transcript.

The total length of DLGAP2 CDNA sequence assembled is approximately $10 \mathrm{~kb}, 2928 \mathrm{bp}$ of which is open reading frame in testis and 3062 bp in brain transcripts (Genbank AF119817 and AF119818). By northern analysis (MTN-1; Clontech) DLGAP2 was found to be expressed mainly in brain (Figure2).

We established the genomic structure of DLGAP2 by sequencing genomic clones obtained from a plasmid library of BAC 156G21 using part of DLGAP2 as a probe, and from 


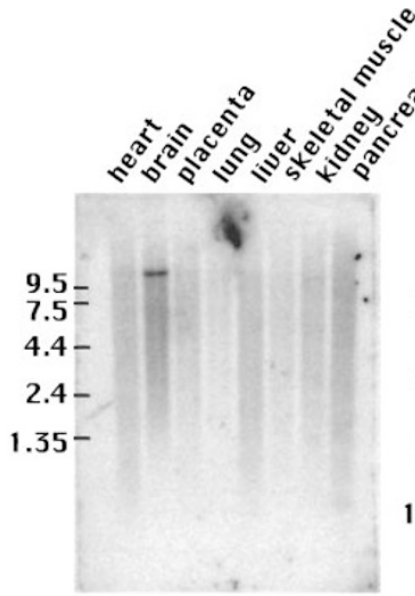

A

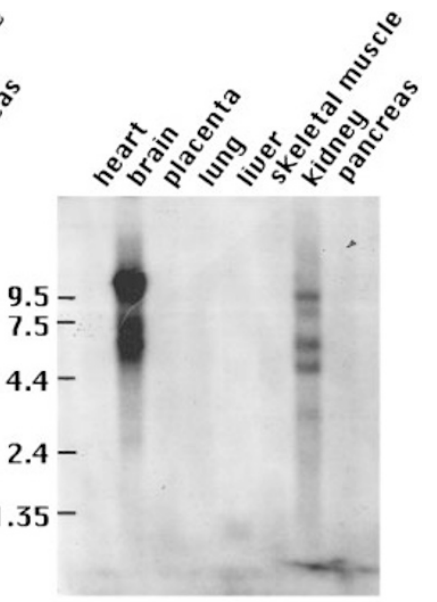

B
Figure 2 The tissue expression pattern of DLGAP2 A Only a $10 \mathrm{~kb}$ brain-specific transcript is detected using a probe from the 3' untranslated region (nt 8059-8626; GenBank AF119817). B Using a probe from the coding region (nt 753-1790; GenBank AF119817) additional smaller brain transcripts, as well kidney transcripts of relatively weak intensity are detected suggesting either alternatively spliced forms of DLGAP2 or hybridisation to homologous DLGAP genes.

sequence data derived from the sequencing effort of the EPMR region (Columbia University Genome Center, New York) as well as from a $120 \mathrm{~kb}$ genomic sequence present in the database (GenBank AC005010). The sequences of the exon-intron boundaries are shown in Table1. We found 12 coding exons that corresponded to the brain transcript (exons2-13, Figure1). The putative $103 \mathrm{bp}$ of coding sequence as well as the $5^{\prime}$ untranslated sequence are still missing from the brain cDNA sequence (see above). Exons 2 and 3 are alternatively spliced in testis, where the last 29 bp
Table 2 Primers used to amplify the coding regions for mutation detection. Primers for exon 2 amplify both exon $2 a$ and $2 \mathrm{~b}$ sequences (see Table 1 )

\begin{tabular}{lll}
\hline No. & Forward primer 5'-3' & Reverse primer 5'-3' \\
\hline exon2 & TGAAGATGTGCAGGGAATGA & GCTAACGTGTGTTGTGGGA \\
exon3 & CCAGCCTGGGCGACAGAG & CGACAATACCACCTGCTGTG \\
exon4 & TCCAAAAAGGAGCTGATGCT & CGCAGGCAGTGGGAAAGT \\
exon5 & GTGTGGGTTGGATGGTATT & AGACTGGACCCCAGGGAG \\
exon6 & GTAACGTGATGGTGACCCTG & TATGCCTCTAGAGTCCCCGC \\
exon7 & AGTAGACCACAGGCTGACGG & TGCTGGGGTTACAGTCAGTG \\
exon8 & CAAAATAGTCCCTTGCCCAG & AAGGACAGGCATGATTGAGG \\
exon9 & TCCTCTCAGAAGGGCTACA & CACTTGAATACCAAAGGGAGGA \\
exon10 & GCTCCCTTGGTGTGATGTTT & AATGATGCCCATTAGCTTGG \\
exon11 & CTCTCCTGGCTCTGAACACC & AGTGAGAAAGGGGGTTTGCT \\
exon12 & AGCTCGCGAGCTGCTGG & GCCACAACCGTCCCCTCG \\
\hline
\end{tabular}

of exon 2 and the first $68 \mathrm{bp}$ of exon 3 form the $5^{\prime}$ untranslated region in addition to the $22 \mathrm{bp}$ of sequence encoded by exon 1 (Figure 1).

We then proceeded to screen the known coding regions and their immediately flanking intronic sequences in DLGAP2 for mutations by sequencing amplified genomic DNA from two patients and one control individual. The primers used for sequencing are shown in Table2. A homozygous silent substitution 1928G $>$ A (both GAG and GAA coding for Glu) was found in exon 6 in the two patients. As an EPMR carrier was also found homozygous for this change it was considered a polymorphism. No other sequence differences between patients and the control were identified.

Based on sequence information obtained from GenBank (AC005010), marker 140CA is located between exons 2 and 3 of DLGAP2 (see Figure1). The region telomeric to 140CA has been previously excluded from harbouring the EPMR mutation on the basis of a recombination event that has occurred with 140CA and markers telomeric to it in one EPM R family. ${ }^{5}$ Using a novel tetranucleotide marker, 85GGAA (GenBank

Table 1 Exon-intron boundaries of the known DLGAP2 gene sequence. The exon and intron sequences are shown in upper case and lower case letters, respectively. The last two columns indicate the nucleotide positions of the exons in the DLGAP2 brain and testis cDNA sequences

\begin{tabular}{|c|c|c|c|c|}
\hline No. & Intron-Exon boundary sequence & Size (bp) & $\begin{array}{l}\text { Position in } \\
\text { brain CDNA }\end{array}$ & $\begin{array}{l}\text { Position in } \\
\text { testis cDNA }\end{array}$ \\
\hline 1 & TTCAGCGGCCTGAATTCTAGAA ${ }^{a}$ & 22 & & $1-22$ \\
\hline $2 a$ & gtctttgcagAGGAAGAAGC....GAGGATCTAGgtagagtaca & 66 & $1-66$ & \\
\hline $2 b$ & cgggcatcagCTTTCCGGGG.....GAGGATCTAGgtagagtaca & 29 & & $23-51$ \\
\hline 3 & ttcCсcacagACCCGCAGTA.... СТACCTCCAGgtaagcaggc & 1058 & $67-1124$ & $52-1109$ \\
\hline 4 & ctgctcccagGTACCTCAGG.....AGCACCAGACgtaagtgaga & 212 & $1125-1336$ & $1110-1321$ \\
\hline 5 & tttcctgtagCCAGACСTAC..... CGTGAGCCAGgtcagggtcC & 148 & 1137-1484 & $1322-1469$ \\
\hline 6 & atgattgcagGTGAGCGAGG... TCAACAGCAGgtaaggggac & 220 & $1485-1704$ & $1470-1689$ \\
\hline 7 & tttcttacagCTGTCTCATA.....CGGGATTCAGgtagctgctc & 350 & $1705-2054$ & $1690-2039$ \\
\hline 8 & ttttgtttagGATTCTGAAT.....AAGGTCAGATgtaagtaccg & 42 & $2055-2096$ & $2040-2081$ \\
\hline 9 & gttgaattagGTGGAAACGG.....ATGAGAAGCGgtaactcagc & 86 & 2097-2182 & $2082-2167$ \\
\hline 10 & ccttttgcagACACGGACGT.... TCGGAGGAAAgtaagagctc & 416 & $2183-2598$ & $2168-2583$ \\
\hline 11 & ttttaaacagTTCTCGGTAA..... АСAGAATATGgtaagtgaat & 92 & $2599-2690$ & $2584-2675$ \\
\hline 12 & gtgtccccagGACCCCAGCG....GGAAAGAAAGgtaagggcat & 153 & $2691-2843$ & $2676-2828$ \\
\hline 13 & tgcttttcagGAAGAAAGAA.... TATTTAGTTM-(A)n & 7290 & 2844-10133 & 2829-10118 \\
\hline
\end{tabular}

aThe exon-intron boundaries of this non-coding exon were not determined. 


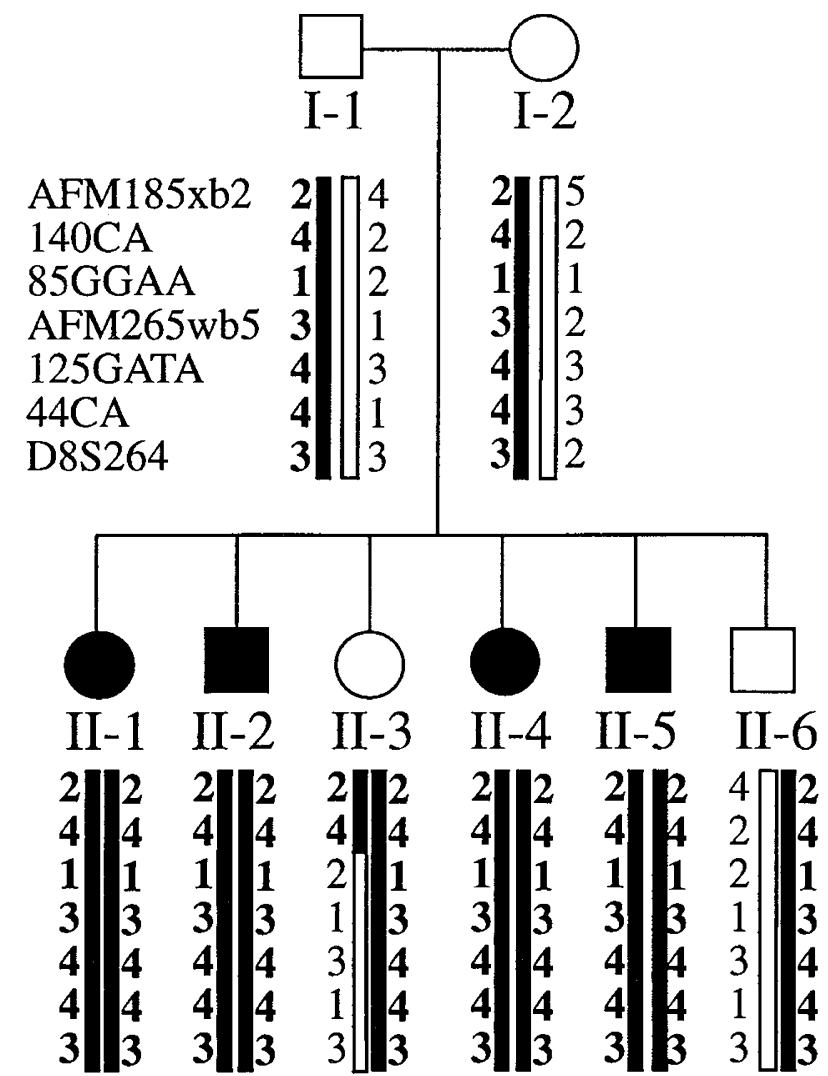

Figure 3 Pedigree of an EPMR family showing a recombination event refining the EPMR critical region telomeric to 140CA. The disease-associated haplotypes are shown with filled boxes. The recombination breakpoint is seen between markers 140CA and 85GGAA in the paternal meiosis in individual II-3.

acc. no AC005010; primers 5'-GGATGC AAG GAT GGAAGG AT-3' and 5'-CCATCC ATC TITAAG ACC CA-3') within the same intron as 140CA, some $7 \mathrm{~kb}$ centromeric to it (Figure 1), this recombination event was further refined between markers 140CA and 85GGAA (Figure3). Thus, based on the localisation of markers 140CA and 85GGAA in relation to DLGAP2 (Figure1), the region 5 ' to exon 3 of the DLGAP2 CDNA was excluded from containing the EPM R mutation.

In this study we describe the characterisation of a novel gene, DLGAP2 localised in the EPMR critical region. Our results provide evidence that DLGAP2 is not the gene underlying EPMR, although as a brain gene interacting with ion channels through the PSD-95 protein DLGAP2 was initially considered a reasonable positional and functional candidate.

\section{Acknowledgements}

SR and YZ contributed equally to this work. Supported by the Academy of Finland, the Sigrid Juselius Foundation, the Ulla Hjelt Foundation, and the Finnish State Grant TYH8310.

\section{References}

1 Hirvasniemi A, Lang $\mathrm{H}$, Lehesjoki A-E, Leisti J: Northern epilepsy syndrome: an inherited childhood onset epilepsy with associated mental deterioration. J Med Genet 1994; 31: 177-182.

2 Haltia M, Tyynelä J, Hirvasniemi A, Herva R, Ranta S, Lehesjoki A-E: CLN8: northern epilepsy. In: Goebel H, Mole SE, Lake BD (eds). The Neuronal Ceroid Lipofuscinoses (Batten's Disease). IOS Press: Amsterdam, 1999; pp117-124.

3 Tahvanainen E, Ranta S, Hirvasniemi A et al: The gene for a recessively inherited human childhood progressive epilepsy with mental retardation maps to the distal short arm of chromosome 8 . Proc Natl Acad Sci USA 1994; 91: 7267-7270.

4 Ranta S, Lehesjoki A-E, Hirvasniemi A et al: Genetic and physical mapping of the progressive epilepsy with mental retardation (EPMR) locus on chromosome 8p. Genome Res 1996; 6: 351-360.

5 Ranta S, Lehesjoki A-E, Bonaldo MF et al: High-resolution mapping and transcript identification at the progressive epilepsy with mental retardation locus on chromosome 8p. Genome Res 1997; 7: 887-896.

6 Satoh $\mathrm{K}$, Yanai $\mathrm{H}$, Senda $\mathrm{T}$ et al: DAP-1, a novel protein that interacts with the guanylate kinase-like domains of hDLG and PSD-95. Genes Cells 1997; 2: 415-424.

7 Takeuchi M, Hata Y, Hirao K, Toyoda A, Irie M, Takai Y: SAPAPS. A family of PSD-95/SAP90-associated proteins localized at postsynaptic density. J Biol Chem 1997; 272: 11943-11951.

$8 \mathrm{Kim}$ E, Naisbitt S, Hsueh Y-P et al: GKAP, a novel synaptic protein that interacts with the gyanylate kinase-like domain of the PSD95/SAP90 family of channel clustering molecules. I Cell Biol 1997; 136: 669-678.

9 Bievert C, Schroeder BC, Kubisch C et al: A potassium channel mutation in neonatal human epilepsy. Science 1998; 279: 403-406.

10 Charlier C, Singh NA, Ryan SG et al: A pore mutation in a novel KQT-like potassium channel gene in an idiopathic epilepsy family. Nat Genet 1998; 18: 53-55.

11 Singh NA, Charlier C, Stauffer D et al: A novel potassium channel gene, KCNQ2, is mutated in an inherited epilepsy of newborns. Nat Genet 1998; 18: 25-29.

12 Schuler GD, Boguski MS, Stewart EA et al: A gene map of the human genome. Science 1996; 274: 540-546. 